CONFORMAL GEOMETRY AND DYNAMICS

An Electronic Journal of the American Mathematical Society

Volume 1, Pages 24-27 (June 19, 1997)

S $1088-4173(97) 00016-7$

\title{
BRANCH SETS OF UNIFORMLY QUASIREGULAR MAPS
}

\author{
G. J. MARTIN
}

\begin{abstract}
Let $n \geq 2$ and $f: \mathbb{S}^{n} \rightarrow \mathbb{S}^{n}$ be a quasiregular mapping with branch set $B_{f}$, the set where $f$ fails to be locally injective. We show that there is a quasiregular mapping $g: \mathbb{S}^{n} \rightarrow \mathbb{S}^{n}$ with $B_{g}=B_{f}$ and such that $g$ can be chosen to be conformal (rational) with respect to some measurable Riemannian structure on $\mathbb{S}^{n}$. Hence $g$ is uniformly quasiregular. That is, $g$ and all its iterates are quasiregular with a uniform bound on the dilatation.
\end{abstract}

\section{INTRODUCTION}

A conformal structure on the $n$-sphere $\mathbb{S}^{n}$ is a bounded measurable map $\mu: \mathbb{S}^{n} \rightarrow$ $S(n)$, the space of symmetric positive definite $n \times n$ matrices of determinant 1 . We view $\mu$ as providing a measurable ellipsoid field on $\mathbb{S}^{n}$ such that the eccentricity of the ellipsoids is uniformly bounded. The structure $\mu \equiv$ Identity is the usual conformal structure of $\mathbb{S}^{n}$. A $\mu$-rational map of $\mathbb{S}^{n}$ is a mapping which is conformal with respect to the structure induced by $\mu$. More precisely, $f: \mathbb{S}^{n} \rightarrow \mathbb{S}^{n}$ is a mapping in the Sobolev class $W^{1, n}\left(\mathbb{S}^{n}, \mathbb{S}^{n}\right)$ whose Jacobian determinant $J_{f}(x)>0$, a.e., and satisfies the first order nonlinear PDE

$$
D f^{t}(x) \mu(f(x)) D f(x)=J_{f}(x)^{2 / n} \mu(x) \quad \text { a.e. } \mathbb{S}^{n},
$$

where $D f$ is the Jacobian matrix. Such a map $f$ is a quasiregular mapping and can be redefined on a set of measure zero so as to be continuous, discrete and open [6]. We refer to Rickman's book [6] for the basics of the theory of quasiregular mappings. The reader should consult [3] for a more detailed discussion of measurable conformal structures. An important feature of a $\mu$-rational mapping is that the mapping is $K$-quasiregular with the dilatation $K$ depending only on the eccentricity of the ellipsoid field and possibly the dimension $n$. Hence $g$ and all its $m$-fold iterates $g^{m}$ (compositions with itself) are quasiregular with a uniform bound on the dilatation. We call such a mapping a uniformly quasiregular mapping following the terminology used in studying groups of quasiconformal mappings since the semigroup $\left\{g^{m}\right\}_{m \geq 1}$ is uniformly quasiregular.

Note that if $\mu$ is the usual conformal structure, the equations of (1.1) reduce to the well known Cauchy-Riemann system. When $n=2$ the solutions are analytic functions of the Riemann sphere $\mathbb{S}^{2}$, and hence are rational mappings. This motivates the terminology " $\mu$-rational". For $n \geq 3$ Gehring's version of the Liouville Theorem ([1], [6]) tells us that for the usual conformal structure the only solutions to (1.1) in the desired Sobolev space are the Möbius transformations, that is, finite

Received by the editors January 5, 1997 and, in revised form, April 16, 1997.

1991 Mathematics Subject Classification. Primary 30C60.

Research supported in part by a grant from the N.Z. Marsden Fund.

(C)1997 American Mathematical Society 
compositions of reflections in spheres and hyperplanes. All solutions in this case are therefore globally injective.

Given a $\mu$-rational map $f$ we define the branch set of $f, B_{f}$, to be that subset of $\mathbb{S}^{n}$ where $f$ fails to be locally injective. Since $f$ is quasiregular, $B_{f}$ is compact.

In [3] the first examples of conformal structures $\mu$ that admit noninjective $\left(B_{f} \neq\right.$ $\emptyset$ ) $\mu$-rational mappings in dimension $n \geq 3$ were given. These examples came as a bit of a surprise. It seemed to be a widely held belief that the fact that all the iterates had a uniform dilatation bound together with known results about the regularity theory of solutions to (1.1) and the growth of dilatation about branch sets as maps wind around them, would have to imply injectivity, and perhaps often a quasiconformal conjugacy to a Möbius transformation. The equivalence of the notion of a uniformly quasiregular mapping and a $\mu$-rational mapping is also shown in [3]. This is done by constructing a measurable conformal structure which is invariant under a uniformly quasiregular semigroup generated by the iterates of one element.

Important new examples by V. Mayer [5] have given further surprises. Mayer constructs $\mu$-rational maps of Lattès type, with completely chaotic dynamics, and associated examples with Julia sets consisting of a codimension one sphere, with a super attracting fixed point. Further examples now exist of quasispheres as Julia sets about attracting fixed points and parabolic fixed points of both standard and nonstandard type. That is, examples for which the classical petal theorem giving local conjugacy to the conformal dynamical model $x \mapsto x+1$ can and cannot hold [4]. See too [2] for a proof of the local quasiconformal conjugacy for attracting fixed points.

It now seems that there is a completely viable and interesting theory of the dynamics associated with the iteration of uniformly quasiregular mappings. The most important unresolved question appears to be the existence or otherwise of rotational dynamics. Are there examples analogous to the holomorphic dynamics yielding Siegel disks?

All the known examples mentioned above have a particularly simple branch set. It was suggested to us that in order for a quasiregular mapping to have all its iterates of uniformly bounded dilatation it was perhaps necessary that the branch set have simple structure. The aim of this note is to show that this is not the case, that is, the branch sets of uniformly quasiregular mappings can be essentially arbitrary.

Theorem 1.2. Let $f: \mathbb{S}^{n} \rightarrow \mathbb{S}^{n}$ be a quasiregular mapping with branch set $B_{f}$. Then there is a quasiregular mapping $g: \mathbb{S}^{n} \rightarrow \mathbb{S}^{n}$ and a conformal structure $\mu$ such that $g$ is a $\mu$-rational mapping with $B_{g}=B_{f}$. In particular $g$ is uniformly quasiregular.

\section{Proof of Theorem 1.2}

Let $f: \mathbb{S}^{n} \rightarrow \mathbb{S}^{n}$ be quasiregular of degree $d<\infty$. Let $x_{0} \in \mathbb{S}^{n}$ have the following properties:

(1) $x_{0}, f\left(x_{0}\right)$ and $f^{-1}\left(x_{0}\right)$ do not meet $B_{f}$.

(2) There is a small ball $U_{0}=B\left(x_{0}, r\right)$ about $x_{0}$ such that $f^{-1}\left(U_{0}\right)$ has components $U_{1}, \ldots, U_{d}$ pairwise disjoint and such that $f: U_{i} \rightarrow U_{0}$ is injective.

(3) $f\left(U_{0}\right)$ is disjoint from $\bigcup_{i=0}^{d} U_{i}$. 
In fact almost every point of $\mathbb{S}^{n}$ has the above properties $[6]$. Let $\left\{x_{1}, \ldots, x_{d}\right\}=$ $f^{-1}\left(x_{0}\right)$ and let $a, b>0$ be so small that $2 b<a$ and

$$
\begin{aligned}
B\left(x_{i}, a\right) & \subset U_{i}, \quad i=0, \ldots, d, \\
B\left(f\left(x_{0}\right), a\right) & \subset f\left(U_{0}\right), \\
B\left(x_{0}, b\right) & \subset \bigcap_{i=1}^{d} f\left(B\left(x_{i}, a\right)\right), \\
f\left(B\left(x_{0}, b\right)\right) & \subset B\left(f\left(x_{0}\right), a\right) .
\end{aligned}
$$

We now define an intermediary map $g_{1}$ as follows. On $\mathbb{S}^{n} \backslash \bigcup_{i=0}^{d} B\left(x_{i}, a\right)$ we set $g_{1}=f$. For $1 \leq i \leq d, g_{1} \mid B\left(x_{i}, b\right)$ is the translation $x \mapsto x-x_{i}+x_{0}$. Next $g_{1} \mid B\left(x_{0}, b\right)$ is the translation $x \mapsto x-x_{0}+f\left(x_{0}\right)$. Finally on the annular regions $g_{1} \mid B\left(x_{i}, a\right) \backslash B\left(x_{i}, b\right)$ is obtained for each $i=0,1, \ldots, d$ by application of Sullivan's version of the Annulus Theorem for quasiconformal mappings [7]. That is, $g_{1} \mid \partial B\left(x_{i}, a\right)=f$ and $g_{1} \mid \partial B\left(x_{i}, b\right)$ is the appropriate translation. The conditions (2.1)-(2.4) imply the map $g_{1}: \mathbb{S}^{n} \rightarrow \mathbb{S}^{n}$ is well defined and quasiregular. Additionally we have by construction $B_{g_{1}}=B_{f}$.

Next let $\Phi$ be a conformal mapping which exchanges $B\left(x_{0}, b\right)$ with its complement and set

$$
g=\Phi \circ g_{1}: \mathbb{S}^{n} \rightarrow \mathbb{S}^{n}
$$

It is clear that $B_{g}=B_{f}$. We first wish to show that $g$ and all its iterates are uniformly quasiregular. This is because we have built a conformal trap as discussed in [3]. We sketch this here. First, if $x \in B=B\left(x_{0}, b\right)$, then $g \mid B$ is conformal and $g(B)=\Phi\left(f\left(x_{0}\right)+B\left(x_{0}, b\right)\right) \subset B$ by (3) and (2.4). Clearly then $g^{m}|B=g \circ \cdots \circ g| B$ is conformal for all $m \geq 1$. Next if $x \in \mathbb{S}^{n} \backslash \bigcup_{i=1}^{d} B\left(x_{i}, b\right)$, then $g_{1}(x) \in \mathbb{S}^{n} \backslash B$ and hence $g(x) \in B$. Therefore $g^{m} \mid \mathbb{S}^{n} \backslash \bigcup_{i=1}^{d} B\left(x_{i}, b\right)$ is quasiregular for each $m$, with a uniform bound on the dilatation by our first observation. Finally, if $x \in B\left(x_{i}, b\right)$, $i=1, \ldots, d$, then $g$ is a conformal translation followed by the conformal mapping $\Phi$. Thus the iterates of $g$ stay conformal at $x$ until it passes into the complement of the balls $B\left(x_{i}, b\right)$. Then it picks up some distortion before passing into $B$ and the iterates again stay conformal. In this way one also sees the Cantor set in $\bigcup_{i=1}^{d} B\left(x_{i}, b\right)$ which forms the Julia set of the mapping $g$. Other aspects of the dynamics of these maps are discussed in [2].

Now the mapping $g$ has the same branch set as $f$ and has the property that the semigroup it generates under composition is cyclic and uniformly quasiregular. In [3] it is shown how one constructs a measurable bounded conformal structure $\mu$ preserved by $g$ (the algebraic restriction is necessary). This completes the proof of Theorem 1.2.

Remark. If one is interested in the bounds on the dilatation one finds that our construction here, together with that in [3], shows that the maximal dilatation of $g$ and also of the invariant conformal structure $\mu$ depends only on the dilatation of $f$ and the dimension $n$. The key places where the dilatation needs to be examined are in the application of the Annulus Theorem (here we have chosen $2 b<a$ so the annular region is thick; the appropriate dilatation estimates are carefully spelled out in [7]) and in the averaging process constructing $\mu$ (estimates on the dilatation in this construction are given in [3]). 


\section{REFERENCES}

[1] F. W. Gehring, Rings and quasiconformal mappings in space, Trans. Amer. Math. Soc. 103 (1962) 353-393. MR 25:3166

[2] A. Hinkkanen and G. J. Martin, Attractors in quasiregular semigroups, Proc. XVI Nevanlinna colloquium, Eds. I. Laine and O. Martio, de Gruyter, Berlin-New York, 1996, 135-141. CMP 97:06

[3] T. Iwaniec and G. J. Martin, Quasiregular semigroups, Ann. Acad. Sci. Fenn. Math. 21 (1996) 241-254. CMP 96:17

[4] G. J. Martin, The dynamics of uniformly quasiregular mappings, to appear.

[5] V. Mayer, Uniformly quasiregular mappings of Lattès type, Preprint.

[6] S. Rickman, Quasiregular mappings, Springer-Verlag 1993. MR 95g:30026

[7] P. Tukia and J. Väisälä, Lipschitz and quasiconformal approximation and extension, Ann. Acad. Sci. Fenn. 6 (1981) 303-342. MR 84a:57016

Department of Mathematics, University of Auckland, Auckland, New Zealand

E-mail address: martin@math.auckland.ac.nz 